\title{
Lipolytic Changes in Fermented Sausages Produced with Turkey Meat: Effects of Starter Culture and Heat Treatment
}

\author{
Betül Karslioğlu, Ümran Ensoy Çiçek*, Nuray Kolsaricii', and Kezban Candoğan ${ }^{1}$ \\ Department of Food Engineering, University of Ankara, 06110, Dişkap1, Ankara, Turkey \\ ${ }^{1}$ Department of Food Engineering, Faculty of Engineering and Natural Sciences, \\ University of Gaziosmanpaşa, Tasliciftlik Campus, 60240 Tokat, Turkey
}

\begin{abstract}
In this study, the effects of two different commercial starter culture mixes and processing methodologies (traditional and heat process) on the lipolytic changes of fermented sausages manufactured with turkey meat were evaluated during processing stages and storage. Free fatty acid (FFA) value increased with fermentation and during storage over $120 \mathrm{~d}$ in all fermented sausage groups produced with both processing methodologies $(p<0.05)$. After drying stage, free fatty acid values of traditional style and heat processed fermented sausages were between $10.54-13.01 \%$ and $6.56-8.49 \%$, respectively. Thiobarbituric acid (TBA) values of traditionally processed fermented sausages were between $0.220-0.450 \mathrm{mg} \cdot \mathrm{kg}^{-1}$, and TBA values of heat processed fermented sausages were in a range of $0.405-0.795 \mathrm{mg} \cdot \mathrm{kg}^{-1}$. Oleic and linoleic acids were predominant fatty acids in all fermented sausages. It was seen that fermented sausage groups produced with starter culture had lower TBA and FFA values in comparison with the control groups, and heat application inhibited the lipase enzyme activity and had an improving effect on lipid oxidation. As a result of these effects, heat processed fermented sausages had lower FFA and higher TBA values than the traditionally processed groups.
\end{abstract}

Key words: traditional fermented sausages, turkey meat, Lactobacillus sake, Pediococcus pentosaceus, lipid oxidation

\section{Introduction}

Traditional Turkish dry fermented sausage can be defined as meat product which is manufactured by chopping and mincing meat and fat with spices, certain additives and starter cultures, then filling into casings and ripening/ drying (Bozkurt and Erkmen, 2007; Çolak et al., 2007; Ensoy et al., 2010; Soyer et al., 2005). Bacterial components of starter cultures are staphylococci and lactic acid bacteria (Casaburi et al., 2007; Drosinos et al., 2005; Leroy et al., 2006; Rantsiou and Cocolin, 2006). The addition of starter cultures shortens the ripening period, ensures the color development, influences flavour and improves the product safety (Ferreira et al., 2006; Leroy et al., 2006; Lücke, 2000).

Lipids generally form the major fraction in fermented sausages that ranges from $25 \%$ to $55 \%$ (Ordonez et al.,

\footnotetext{
*Corresponding author: Ümran Ensoy Çiçek, Department of Food Engineering, Faculty of Engineering and Natural Sciences, University of Gaziosmanpaşa, Tasliciftlik Campus, 60240 Tokat, Turkey. Tel: 90-356-252-1616, Fax: 90-356-252-1729, E-mail: umran.ensoy@gop.edu.tr
}

1999) and the changes in lipid fractions during sausage processing have a major impact, both desirable and deleterious for final product quality (Talon et al., 2000; Visessanguan et al., 2006). The flavours of these products were found to be related to hydrolytic and oxidative changes occurring in the lipid fraction during ripening (Chizzolini et al., 1998; Fernandez et al., 2000; Molly et al., 1996; Ordonez et al., 1999).

Hydrolysis, the partial degradation of the lipid fraction in fermented sausages, is one of the main secondary biochemical processes, which occurs as a consequence of the action of glycerol ester hydrolases (Molly et al., 1996; Nassu et al., 2003; Ordonez et al., 1999; Samelis et al., 1993). These enzymes are derived from both muscle tissue and microorganisms (Chizzolini et al., 1998; Demeyer et al., 1974; Molly et al., 1996; Ordonez et al., 1999; Visessangun et al., 2006). Although micrococci-staphylococci are the main lipolytic microorganisms, bacterial contribution to lipolysis is judged to be limited, but variable in relation with different manufacturing parameters e.g., temperature, $\mathrm{pH}$ and time of processing meat (Chizzolini et al., 1998; Samelis et al., 1993; Visessanguan et al., 2006). The enzyme activity resulted in liberation of 
fatty acids which undergo oxidative processes yielding lipid peroxides and carbonyls (Ordonez et al., 1999; Visessanguan et al., 2006). Lipid oxidation is one of the primary mechanisms of quality deterioration in meat products (Nassu et al., 2003; Talon et al., 2000). The excess of oxidation could reach the level of rancidity resulting in an unacceptable product for human consumption (Zanardi et al., 2002). Additionally, during sausage manufacturing several factors influence the rate of lipid oxidation such as type (poultry, beef, pork) and composition of meat, grinding, smoking, heat treatment, temperature, starter culture and adding exogenous components (salt, nitrite, spices, antioxidants) (Talon et al., 2000).

Turkey meat, an inexpensive source of animal protein contains low levels of cholesterol and total lipids and has a high level of polyunsaturated fatty acids (Baggio et al., 2002; Deumier and Collignan 2003; Ferreira et al., 2000; Wong and Sampugna, 1993). As a result of these reasons, turkey meat has been utilized in manufacturing of a variety of meat products such as fermented sausages, as a substitute for red meats (Ferreira et al., 2000). In Turkey, many facilities use turkey meat as a main raw material or with beef meat in the formulation of meat products. Heat treatment also became a common process stage of fermented sausage production which is utilized after fermentation or a short drying stage. The effects of different starter cultures and heat treatments on the properties of fermented beef sausages were investigated by many researchers, but the effects of commercial starter culture and the heat treatment on the chemical properties of fermented turkey sausages were not investigated. The aim of this research was to gain the effects of both commercial starter culture mixes (Lactobacilli/Staphylococci and Lactobacilli/Staphylococci/Pediococci) and heat treatment on the lipolytic changes of fermented turkey sausages during ripening and refrigerated storage for four months.

\section{Materials and Methods}

\section{Sausage production}

Two experiments were carried out involving three groups of fermented sausage. Turkey thigh meat and sheep-tail fat were ground using a plate with $12 \mathrm{~mm}$ orifices using a meat grinder (Ari Torna, Turkey). Then, other ingredients were added as follows; commercial sausage spice mix (Bayramoğlu-Pabay Chemical Material Marketing and Distribution, Turkey), $\mathrm{NaCl}$, sodium nitrite and sodium nitrate (food grade, Merck, Germany). Two of the fermented sausage batches were inoculated with freeze dried starter cultures Texel (Rhöne Poulenc, Courbevoie Cedex, France) (named S1) or with freeze dried Biobak K (Wiberg, Salzburg, Austria) (named S2) as a second starter culture. Texel contained Lactobacillus sake, Staphylococcus carnosus, Staphylococcus xylosus II, and Biobak K contained Lactobacillus sake, Staphylococcus xylosus and Pediococcus pentosaceus. These starter cultures were obtained from different large meat facilities in Turkey. The preparation of these starter cultures was completed using the respective manufacturer's instructions. The third fermented sausage group was not inoculated with any commercial starter culture mix and manufactured as a control group (named C).

After combining the ingredients with the meat and sheep tail fat using a Yuneka mixer (Yuneka Metal, Turkey), the fermented sausage mixtures were reground through a plate with $3 \mathrm{~mm}$ orifices, stored overnight at $4^{\circ} \mathrm{C}$ (initial mix), and then stuffed into natural beef intestines using a hydraulic filling machine (Yuneka Metal, Turkey). Then, fermented sausage chubs were hung on stainless steel hangers, allowed to equilibrate at $18^{\circ} \mathrm{C}$ for $6 \mathrm{~h}$, and placed in a Biogen SCK $4500 \times 1$ model ripening chamber (Biogen, Turkey) equipped with a process control system for $48 \mathrm{~h}$ using a following programmed fermentation schedule: (step 1) at $26^{\circ} \mathrm{C}$ and $95-98 \%$ relative humidity (RH) for $36 \mathrm{~h}$, (step 2) at $23^{\circ} \mathrm{C}$ and $80 \% R H$ for $12 \mathrm{~h}$.

After fermentation, fermented sausage chubs from each group were randomly subdivided into two parts. One group was placed in a drying room, and dried at $22^{\circ} \mathrm{C}$ and $50-55 \% \mathrm{RH}$ for $2 \mathrm{~d}$ then at $18^{\circ} \mathrm{C}$ and $50-55 \% \mathrm{RH}$ for $5 \mathrm{~d}$ (total $7 \mathrm{~d}$ ) (T: traditional style processing without heat application). The other group was heat processed following the fermentation stage in a Biogen Oven programmed to $70^{\circ} \mathrm{C}$ until an internal temperature of approximately $55^{\circ} \mathrm{C}$ was attained, and then kept at this temperature for $5 \mathrm{~min}$ ( $\mathrm{H}$ : heated fermented sausages). Internal temperature of the fermented sausage was measured using Microprocessor Digital Thermometer with Type K thermo-couple (Hanna Instruments, Deutschland, Germany). Following heating, the fermented sausage were cooled with tap water, surface dried, placed in a drying room, and dried for $7 \mathrm{~d}$ using the same drying conditions indicated above. After drying, each chub was vacuum-packed individually, and stored in refrigerated conditions for four months for further analysis.

\section{Sampling}

Two randomly selected chubs were removed at each stage of processing such as initial mix, fermentation, heating and 
drying. For storage periods, samples were taken on the first day (day 0), day 30, day 60, day 90 and day 120 . Free fatty acid content and thiobarbituric acid (TBA) value were evaluated at all steps mentioned above. Fatty acid compositions of fermented sausages were analyzed after initial mix, and during storage periods given above. In addition, moisture, fat, protein, ash and salt contents of fermented sausages were measured on the day 0 .

\section{Compositional analysis}

In order to prepare the samples for analysis, after removing the outer casing, samples were thoroughly cut up and ground with a food grinder (Bosch, Germany) until a homogeneous sample was obtained. Moisture, protein $(\mathrm{N}$ $\times 6.25$ ), fat and ash contents were determined in duplicate following AOAC (2006) methods numbered as 950.46, $928.08,960.39$ and 950.13 , respectively. Salt content of fermented sausages was measured according to Lees (1975).

\section{Free fatty acid (FFA) content}

The lipids were extracted from $100 \mathrm{~g}$ of samples with a solvent mixture of chloroform:methanol (2:1) using a sample:solvent ratio of 1:2 (Bligh and Dyer 1959). FFA content was determined according to AOAC method numbered as 940.28 (AOAC, 2006). Five grams of extracted lipid samples were dissolved in $50 \mathrm{ml}$ of neutral ethyl alcohol, added phenolphthalein indicator and titrated with $0.1 \mathrm{~mol} \mathrm{l}^{-1}$ sodium hydroxide solutions to gain pink color. The result was expressed as oleic acid in percent.

\section{Thiobarbituric acid (TBA) value}

Thiobarbituric acid value was determined as milligram of malondialdehyde per kilogram of sample using the method of Tarladgis et al. (1960). The optical density of the samples was read against the blank at a wavelength of $538 \mathrm{~nm}$ using a UNICAM UV/Vis spectrophotometer (Thermo Scientific, Italy). The readings were multiplied by the factor of 7.8 to convert to milligrams of MA per kilogram of sample.

\section{Fatty acid composition}

To prepare the methyl esters of the fatty acids according to IUPAC (1987), $0.2 \mathrm{~g}$ of lipids obtained at each of the sampling intervals previously noted, were added to 10 $\mathrm{ml}$ of hexane and $0.5 \mathrm{ml}$ of $2 \mathrm{~mol} \mathrm{l}^{-1} \mathrm{KOH}$ solution prepared with methanol. Fatty acid composition of the fermented sausage samples were analyzed using a Shimadzu GC-2010 gas chromatograph (Shimadzu, Japan) equipped with a DB-23 column $(30 \mathrm{~m} \times 0.25 \mathrm{~mm}$ i.d. and $0.25 \mu \mathrm{m}$ film thickness) (Agilent Technologies, USA). Injector, column and detector temperatures were $230^{\circ} \mathrm{C}, 190^{\circ} \mathrm{C}$ and $240^{\circ} \mathrm{C}$, respectively. Split ratio was $80: 1$. Carrier gas was helium at $1.0 \mathrm{ml} \cdot \mathrm{min}^{-1}$ ratio.

\section{Statistical analysis}

All experimental data collected during the processing steps were examined statistically using analysis of variance (ANOVA). A two factorial design and of storage periods were evaluated by applying ANOVA according to a three factorial design with repeated measurements in time. If required, Duncan multiple comparison test was performed to investigate the differences between mean values.

\section{Results}

\section{Compositional analysis}

The effects of different processing methodologies and starter culture utilization on the proximate composition of fermented turkey sausages were not found to be significant $(p>0.05)$. Moisture, protein, fat, ash and salt contents of traditional style fermented sausages were in the range of $46.4-54.7 \%, 21.1-25.3 \%, 20.6-21.6 \%, 3.9-4.7 \%$ and $3.5-4.4 \%$, respectively (Table 1 ). For heated fermented sausages, moisture, protein, fat, ash and salt contents were measured between $41.1-48.3 \%, 25.2-27.4 \%, 22.3-25.6 \%$, $4.3-4.7 \%$ and $4.2-4.7 \%$, respectively (Table 1 ).

\section{Free fatty acid (FFA) content}

FFA contents of fermented sausages produced by both

Table 1. Chemical composition of fermented turkey sausages (\%)

\begin{tabular}{cccccc}
\hline \hline Groups & Moisture & Protein & Fat & Ash & Salt \\
\hline C-T & $54.7 \pm 3.5$ & $21.0 \pm 0.2$ & $21.6 \pm 1.2$ & $3.9 \pm 0.4$ & $3.5 \pm 0.2$ \\
S1-T & $46.4 \pm 0.9$ & $25.2 \pm 0.0$ & $21.1 \pm 1.6$ & $4.6 \pm 0.0$ & $4.4 \pm 0.3$ \\
S2-T & $50.8 \pm 5.4$ & $24.4 \pm 1.7$ & $20.6 \pm 2.2$ & $4.1 \pm 0.2$ & $3.8 \pm 0.0$ \\
C-H & $48.3 \pm 3.3$ & $25.6 \pm 0.9$ & $22.2 \pm 2.0$ & $4.3 \pm 0.2$ & $4.2 \pm 0.0$ \\
S1-H & $41.0 \pm 3.8$ & $27.4 \pm 3.7$ & $25.6 \pm 0.0$ & $4.7 \pm 0.6$ & $4.7 \pm 0.3$ \\
S2-H & $46.4 \pm 1.7$ & $25.2 \pm 0.4$ & $23.1 \pm 0.8$ & $4.5 \pm 0.5$ & $4.4 \pm 0.2$ \\
\hline
\end{tabular}

* Data are the mean \pm standard deviation. 
Table 2. Free fatty acid contents of traditionally processed fermented turkey sausages at various processing stages* (oleic acid\%)

\begin{tabular}{|c|c|c|c|}
\hline \multirow{2}{*}{ Groups } & \multicolumn{3}{|c|}{ Processing Stages } \\
\hline & Initial mix $^{C}$ & Fermentation $^{\mathrm{B}}$ & Drying $^{A}$ \\
\hline$C-T^{a}$ & $1.77 \pm 0.40^{\mathrm{a}}$ & $5.88 \pm 0.56^{\mathrm{a}}$ & $11.10 \pm 3.16^{\mathrm{a}}$ \\
\hline $\mathrm{S} 1-\mathrm{T}^{\mathrm{a}}$ & $1.97 \pm 0.33^{\mathrm{a}}$ & $5.03 \pm 0.21^{\mathrm{b}}$ & $10.54 \pm 2.64^{\mathrm{a}}$ \\
\hline $\mathrm{S} 2-\mathrm{T}^{\mathrm{a}}$ & $1.99 \pm 0.48^{\mathrm{a}}$ & $5.60 \pm 0.25^{\mathrm{ab}}$ & $13.01 \pm 3.92^{\mathrm{a}}$ \\
\hline
\end{tabular}

* Data are the mean \pm standard deviation.

${ }^{\mathrm{a}, \mathrm{b}}$ Means in a column not having a common superscript letter are different $(p<0.05)$.

${ }^{\mathrm{A}-\mathrm{C}}$ Means in a row not having a common superscript letter are different $(p<0.05)$.

traditional and heat processes are given in Table 2. FFA contents of all fermented sausage groups produced either by traditional or heated methodologies were affected by ripening period $(p<0.05)$. The FFA contents of initial mixes expressed as oleic acid (1.8-2.0\%) increased to 5.0$5.8 \%$ after fermentation in both manufacturing methods $(p<0.05)$. Traditional style fermented sausages had final FFA content of $11.1 \%, 10.5 \%$ and $13.0 \%$ for C-T, S1-T and S2-T, respectively. The highest FFA content was observed in traditional style S2-T group which was produced with the starter culture mix containing $L$. sake, S. xylosus and $P$. pentosaceus $(p>0.05)$. The lowest FFA was measured in S1-T group which had the lowest $\mathrm{pH}$ and highest titratable acidity value $(p>0.05)$ (data not shown).

For heated fermented sausages, each processing stage had significant effects on FFA contents $(p<0.05$, Table 3$)$. After drying, FFA values of C-H, S1-H and S2-H were
Table 5. Thiobarbituric acid (TBA) values of traditionally processed fermented turkey sausages at various processing stages*

\begin{tabular}{|c|c|c|c|}
\hline \multirow{2}{*}{ Groups } & \multicolumn{3}{|c|}{ Processing Stages } \\
\hline & Initial mix ${ }^{B}$ & Fermentation $^{B}$ & Drying $^{A}$ \\
\hline $\mathrm{C}-\mathrm{T}^{\mathrm{a}}$ & $0.22 \pm 0.12^{\mathrm{a}}$ & $0.26 \pm 0.06^{\mathrm{a}}$ & $0.41 \pm 0.10^{\mathrm{a}}$ \\
\hline $\mathrm{S} 1-\mathrm{T}^{\mathrm{b}}$ & $0.17 \pm 0.08^{\mathrm{a}}$ & $0.21 \pm 0.10^{\mathrm{a}}$ & $0.22 \pm 0.03^{\mathrm{b}}$ \\
\hline $\mathrm{S} 2-\mathrm{T}^{\mathrm{ab}}$ & $0.23 \pm 0.14^{\mathrm{a}}$ & $0.18 \pm 0.08^{\mathrm{a}}$ & $0.43 \pm 0.06^{\mathrm{a}}$ \\
\hline
\end{tabular}

*Data are the mean \pm standard deviation. TBA values are expressed as milligrams of malondialdehyde per kilogram of sample.

${ }^{\mathrm{a}, \mathrm{b}}$ Means in a column not having a common superscript letter are different $(p<0.05)$.

$\mathrm{A}, \mathrm{B}$ Means in a row not having a common superscript letter are different $(p<0.05)$.

8.49, 7.06, and 6.56 oleic acid \%, respectively. S1-H and S2-H groups had lower FFA contents in comparison with C-H group $(p<0.05)$.

Storage period did have an increasing effect $(p<0.05)$ on FFA contents in both traditional and heat processed fermented sausages, especially after day 60 (Table 4). Traditionally processed $\mathrm{C}-\mathrm{T}$ and $\mathrm{S} 2-\mathrm{T}$ groups had the highest FFA contents among the fermented sausage groups over storage $(p<0.05)$.

\section{Thiobarbituric acid (TBA) value}

The TBA values of both traditional and heat processed fermented sausages observed at processing stages are given in Table 5. The TBA values of initial mixes were between $0.173-0.230 \mathrm{mg} \cdot \mathrm{kg}^{-1}$ samples, for traditionally processed fermented sausages, TBA values reached to 0.415 ,

Table 3. Free fatty acid contents of heat processed fermented turkey sausages at various processing stages* (oleic acid\%)

\begin{tabular}{|c|c|c|c|c|}
\hline \multirow{2}{*}{ Groups } & \multicolumn{4}{|c|}{ Processing Stages } \\
\hline & Initial $\operatorname{mix}^{\mathrm{D}}$ & Fermentation $^{\mathrm{C}}$ & Heating $^{\mathrm{B}}$ & Drying $^{A}$ \\
\hline $\mathrm{C}-\mathrm{H}^{\mathrm{a}}$ & $1.77 \pm 0.40^{\mathrm{a}}$ & $5.88 \pm 0.56^{\mathrm{a}}$ & $6.96 \pm 1.19^{\mathrm{a}}$ & $8.49 \pm 0.08^{a}$ \\
\hline $\mathrm{S} 1-\mathrm{H}^{\mathrm{b}}$ & $1.97 \pm 0.33^{\mathrm{a}}$ & $5.03 \pm 0.21^{\mathrm{b}}$ & $5.88 \pm 0.46^{\mathrm{a}}$ & $7.06 \pm 1.32^{b}$ \\
\hline $\mathrm{S} 2-\mathrm{H}^{\mathrm{b}}$ & $1.99 \pm 0.48^{\mathrm{a}}$ & $5.60 \pm 0.25^{\mathrm{ab}}$ & $6.01 \pm 0.18^{\mathrm{a}}$ & $6.56 \pm 0.53^{b}$ \\
\hline
\end{tabular}

* Data are the meantstandard deviation.

${ }^{\mathrm{a}, \mathrm{b}}$ Means in a column not having a common superscript letter are different $(p<0.05)$.

${ }^{A-D}$ Means in a row not having a common superscript letter are different $(p<0.05)$.

Table 4. Free fatty acid contents of fermented turkey sausages during $120 \mathrm{~d}$ storage* (oleic acid\%)

\begin{tabular}{cccccc}
\hline \hline \multirow{2}{*}{ Groups } & \multicolumn{5}{c}{ Storage Periods } \\
\cline { 2 - 6 } & $0^{\mathrm{C}}$ & $30^{\mathrm{C}}$ & $60^{\mathrm{B}}$ & $90^{\mathrm{B}}$ & $120^{\mathrm{A}}$ \\
\hline $\mathrm{C}-\mathrm{T}^{\mathrm{a}}$ & $11.10 \pm 3.16^{\mathrm{ab}}$ & $17.26 \pm 2.88^{\mathrm{a}}$ & $21.70 \pm 6.96^{\mathrm{a}}$ & $23.43 \pm 9.13^{\mathrm{a}}$ & $26.64 \pm 10.36^{\mathrm{a}}$ \\
$\mathrm{S} 1-\mathrm{T}^{\mathrm{b}}$ & $10.54 \pm 2.64^{\mathrm{abc}}$ & $9.12 \pm 2.09^{\mathrm{bc}}$ & $12.20 \pm 1.88^{\mathrm{bc}}$ & $13.64 \pm 3.22^{\mathrm{b}}$ & $14.86 \pm 4.08^{\mathrm{c}}$ \\
$\mathrm{S} 2-\mathrm{T}^{\mathrm{a}}$ & $13.01 \pm 3.92^{\mathrm{a}}$ & $12.40 \pm 4.59^{\mathrm{b}}$ & $17.70 \pm 5.77^{\mathrm{ab}}$ & $22.33 \pm 6.02^{\mathrm{a}}$ & $24.88 \pm 10.21^{\mathrm{ab}}$ \\
$\mathrm{C}-\mathrm{H}^{\mathrm{b}}$ & $8.49 \pm 0.08^{\mathrm{bcd}}$ & $10.32 \pm 0.34^{\mathrm{bc}}$ & $12.39 \pm 0.82^{\mathrm{bc}}$ & $14.80 \pm 1.80^{\mathrm{b}}$ & $16.02 \pm 0.76^{\mathrm{bc}}$ \\
$\mathrm{S} 1-\mathrm{H}^{\mathrm{c}}$ & $7.06 \pm 1.32^{\mathrm{cd}}$ & $8.23 \pm 0.25^{\mathrm{c}}$ & $8.71 \pm 0.80^{\mathrm{c}}$ & $8.48 \pm 0.98^{\mathrm{b}}$ & $12.12 \pm 1.66^{\mathrm{c}}$ \\
$\mathrm{S} 2-\mathrm{H}^{\mathrm{bc}}$ & $6.56 \pm 0.53^{\mathrm{d}}$ & $8.46 \pm 0.61^{\mathrm{c}}$ & $12.26 \pm 0.23^{\mathrm{bc}}$ & $9.62 \pm 1.83^{\mathrm{b}}$ & $13.86 \pm 0.86^{\mathrm{c}}$ \\
\hline
\end{tabular}

* Data are the meantstandard deviation.

${ }^{\mathrm{a}-\mathrm{c}}$ Means in a column not having a common superscript letter are different $(p<0.05)$.

${ }^{\mathrm{A}-\mathrm{C}}$ Means in a row not having a common superscript letter are different $(p<0.05)$. 
Table 6. Thiobarbituric acid (TBA) values of heat processed fermented turkey sausages at various processing stages*

\begin{tabular}{ccccc}
\hline \hline \multirow{2}{*}{ Groups } & \multicolumn{4}{c}{ Processing Stages } \\
\cline { 2 - 5 } & Initial mix $^{\mathrm{B}}$ & Fermentation $^{\mathrm{B}}$ & Heating $^{\mathrm{B}}$ & Drying $^{\mathrm{A}}$ \\
\hline C-H & $0.22 \pm 0.12$ & $0.26 \pm 0.06$ & $0.27 \pm 0.08$ & $0.43 \pm 0.00$ \\
S1-H & $0.17 \pm 0.08$ & $0.21 \pm 0.10$ & $0.19 \pm 0.06$ & $0.40 \pm 0.05$ \\
S2-H & $0.23 \pm 0.14$ & $0.18 \pm 0.08$ & $0.17 \pm 0.09$ & $0.41 \pm 0.14$ \\
\hline
\end{tabular}

*Data are the mean \pm standard deviation. TBA values are expressed as milligrams of malondialdehyde per kilogram of sample.

${ }^{\mathrm{A}, \mathrm{B}}$ Means in a row not having a common superscript letter are different $(p<0.05)$.

Table 7. Thiobarbituric acid (TBA) values of heat processed fermented turkey sausages during $120 \mathrm{~d}$ storage*

\begin{tabular}{|c|c|c|c|c|c|}
\hline \multirow{2}{*}{ Groups } & \multicolumn{5}{|c|}{ Storage Periods } \\
\hline & $0^{\mathrm{C}}$ & $30^{\mathrm{BC}}$ & $60^{\mathrm{A}}$ & $90^{\mathrm{B}}$ & $120^{\mathrm{A}}$ \\
\hline$C-T^{\text {cd }}$ & $0.41 \pm 0.10^{\mathrm{a}}$ & $0.38 \pm 0.12^{\mathrm{c}}$ & $0.32 \pm 0.15^{\mathrm{c}}$ & $0.33 \pm 0.08^{b c}$ & $0.50 \pm 0.34^{\mathrm{bc}}$ \\
\hline $\mathrm{S} 1-\mathrm{T}^{\mathrm{d}}$ & $0.22 \pm 0.03^{b}$ & $0.32 \pm 0.19^{c}$ & $0.13 \pm 0.01^{\mathrm{c}}$ & $0.20 \pm 0.05^{\mathrm{c}}$ & $0.20 \pm 0.09^{\mathrm{c}}$ \\
\hline $\mathrm{S} 2-\mathrm{T}^{\mathrm{d}}$ & $0.43 \pm 0.06^{\mathrm{a}}$ & $0.22 \pm 0.05^{\mathrm{c}}$ & $0.30 \pm 0.16^{\mathrm{c}}$ & $0.26 \pm 0.12^{b c}$ & $0.31 \pm 0.15^{\mathrm{c}}$ \\
\hline $\mathrm{C}-\mathrm{H}^{\mathrm{a}}$ & $0.43 \pm 0.00^{\mathrm{a}}$ & $1.06 \pm 0.01^{\mathrm{a}}$ & $2.09 \pm 0.68^{\mathrm{a}}$ & $1.62 \pm 0.28^{\mathrm{a}}$ & $2.06 \pm 0.69^{\mathrm{a}}$ \\
\hline $\mathrm{S} 1-\mathrm{H}^{\mathrm{c}}$ & $0.40 \pm 0.05^{\mathrm{a}}$ & $0.41 \pm 0.09^{c}$ & $0.70 \pm 0.24^{\mathrm{bc}}$ & $0.52 \pm 0.18^{\mathrm{b}}$ & $0.66 \pm 0.35^{b c}$ \\
\hline $\mathrm{S} 2-\mathrm{H}^{\mathrm{b}}$ & $0.41 \pm 0.14^{\mathrm{a}}$ & $0.64 \pm 0.02^{b}$ & $1.15 \pm 0.68^{b}$ & $0.37 \pm 0.02^{b c}$ & $1.02 \pm 0.14^{\mathrm{b}}$ \\
\hline
\end{tabular}

*Data are the mean \pm standard deviation. TBA values are expressed as milligrams of malondialdehyde per kilogram of sample.

${ }^{\mathrm{a}-\mathrm{d}}$ Means in a column not having a common superscript letter are different $(p<0.05)$.

${ }^{\mathrm{A}-\mathrm{C}}$ Means in a row not having a common superscript letter are different $(p<0.05)$.

Table 8. Saturated fatty acid (SFA) contents of fermented turkey sausages during $120 \mathrm{~d}$ storage*

\begin{tabular}{|c|c|c|c|c|c|c|}
\hline \multirow{2}{*}{ Groups } & \multicolumn{6}{|c|}{ Periods } \\
\hline & Initial mix $^{\mathrm{B}}$ & $0^{\mathrm{B}}$ & $30^{\mathrm{A}}$ & $60^{A}$ & $90^{A}$ & $120^{\mathrm{A}}$ \\
\hline $\mathrm{C}-\mathrm{T}^{\mathrm{a}}$ & $18.58 \pm 0.41^{b}$ & $18.45 \pm 0.82^{\mathrm{a}}$ & $21.20 \pm 1.17^{\mathrm{a}}$ & $20.23 \pm 0.10^{\mathrm{a}}$ & $21.04 \pm 0.45^{\mathrm{a}}$ & $22.80 \pm 1.27^{\mathrm{a}}$ \\
\hline $\mathrm{S} 1-\mathrm{T}^{\mathrm{a}}$ & $18.30 \pm 0.07^{\mathrm{b}}$ & $18.67 \pm 1.41^{\mathrm{a}}$ & $22.24 \pm 1.01^{\mathrm{a}}$ & $21.42 \pm 2.10^{\mathrm{a}}$ & $22.22 \pm 0.10^{\mathrm{a}}$ & $21.83 \pm 1.55^{\mathrm{a}}$ \\
\hline $\mathrm{S} 2-\mathrm{T}^{\mathrm{a}}$ & $21.10 \pm 1.02^{\mathrm{a}}$ & $14.83 \pm 5.07^{\mathrm{a}}$ & $22.43 \pm 0.99^{\mathrm{a}}$ & $22.64 \pm 1.05^{\mathrm{a}}$ & $21.95 \pm 2.10^{\mathrm{a}}$ & $20.41 \pm 2.39^{a}$ \\
\hline $\mathrm{C}-\mathrm{H}^{\mathrm{a}}$ & $18.58 \pm 0.41^{b}$ & $19.43 \pm 2.26^{\mathrm{a}}$ & $21.74 \pm 2.67^{\mathrm{a}}$ & $21.39 \pm 0.52^{\mathrm{a}}$ & $20.31 \pm 0.82^{\mathrm{a}}$ & $20.54 \pm 1.68^{a}$ \\
\hline $\mathrm{S} 1-\mathrm{H}^{\mathrm{a}}$ & $18.30 \pm 0.07^{b}$ & $19.02 \pm 2.29^{\mathrm{a}}$ & $21.51 \pm 1.71^{\mathrm{a}}$ & $20.80 \pm 3.06^{\mathrm{a}}$ & $22.97 \pm 3.63^{\mathrm{a}}$ & $19.92 \pm 1.34^{\mathrm{a}}$ \\
\hline $\mathrm{S} 2-\mathrm{H}^{\mathrm{a}}$ & $21.10 \pm 1.02^{\mathrm{a}}$ & $17.13 \pm 2.26^{\mathrm{a}}$ & $20.21 \pm 0.41^{\mathrm{a}}$ & $21.47 \pm 1.33^{\mathrm{a}}$ & $23.15 \pm 1.90^{\mathrm{a}}$ & $20.18 \pm 0.36^{\mathrm{a}}$ \\
\hline
\end{tabular}

* Data are the meantstandard deviation.

${ }^{a, b}$ Means in a column not having a common superscript letter are different $(p<0.05)$.

${ }^{\mathrm{A}, \mathrm{B}}$ Means in a row not having a common superscript letter are different $(p<0.05)$.

0.220 and $0.450 \mathrm{mg} \cdot \mathrm{kg}^{-1}$ sample at the end of ripening, for C-T, S1-T and S2-T groups, respectively $(p<0.05)$. The TBA values observed in heated fermented sausages showed a slight increase after heating stage ( $p>0.05$, Table 6$)$. At the end of ripening period, TBA values of heated $\mathrm{C}-\mathrm{H}$, $\mathrm{S} 1-\mathrm{H}$ and S2-H groups were increased to $0.795,0.405$ and $0.413 \mathrm{mg} \cdot \mathrm{kg}^{-1}$ sample, respectively $(p<0.05)$.

Refrigerated storage had significant effect on TBA values of all fermented sausage groups produced by either traditional or heat processes $(p<0.05)$. However, for all treatments, TBA values did not continue to increase throughout storage. During refrigerated storage, heated fermented sausages had higher TBA values than traditional style fermented sausages $(p<0.05$, Table 7$)$. With respect to the lipid oxidation evaluation, different TBA values were observed among the fermented sausage groups, the highest TBA value was measured in $\mathrm{C}-\mathrm{H}$, while the lowest TBA values determined from S1-T and S2-T groups $(p<0.05)$.

\section{Fatty acid composition}

Total SFA, MUFA and PUFA amounts of initial mix of $\mathrm{C}$ groups were determined as $18.5 \%, 43.1 \%$ and $26.0 \%$, respectively. The inital mix of S1 had the percentage of $18.3 \%$ for total SFA, $42.0 \%$ for MUFA and $25.5 \%$ for PUFA. The total SFA, MUFA and PUFA contents of S2 were observed as $21.1 \%, 42.5 \%$ and $28.8 \%$, respectively (Table 8, 9 and 10). In comparison with $\mathrm{C}$ and $\mathrm{S} 1$ sausage mixes, the initial mix of $\mathrm{S} 2$ had the highest total SFA while the initial mix of S1 had the lowest PUFA content $(p<0.05)$. For traditionally processed fermented sausages, sum of SFA in all groups showed slight decreases after drying stage $(p>0.05)$.

The fatty acid compositions of initial mixes and fermented sausages during refrigerated storage for $120 \mathrm{~d}$ were analyzed (data not shown). Oleic, linoleic, palmitic, linolenic and myristic acids were the main fatty acids in fermented sausage samples produced by both processing 
Table 9. Monounsaturated fatty acid (MUFA) contents of fermented turkey sausages during $120 \mathrm{~d}$ storage*

\begin{tabular}{|c|c|c|c|c|c|c|}
\hline \multirow{2}{*}{ Groups } & \multicolumn{6}{|c|}{ Periods } \\
\hline & Initial mix $^{B}$ & $0^{A}$ & $30^{A}$ & $60^{A}$ & $90^{\mathrm{A}}$ & $120^{\mathrm{A}}$ \\
\hline $\mathrm{C}-\mathrm{T}^{\mathrm{b}}$ & $43.23 \pm 1.19^{\mathrm{a}}$ & $44.56 \pm 3.33^{\mathrm{a}}$ & $43.51 \pm 0.26^{\mathrm{a}}$ & $43.78 \pm 1.25^{b}$ & $44.80 \pm 1.33^{\mathrm{a}}$ & $45.20 \pm 2.51^{\mathrm{a}}$ \\
\hline $\mathrm{S} 1-\mathrm{T}^{\mathrm{ab}}$ & $42.08 \pm 0.98^{\mathrm{a}}$ & $43.74 \pm 3.23^{\mathrm{a}}$ & $47.87 \pm 4.65^{\mathrm{a}}$ & $47.43 \pm 1.59^{\mathrm{ab}}$ & $47.77 \pm 1.26^{\mathrm{a}}$ & $46.09 \pm 2.46^{\mathrm{a}}$ \\
\hline $\mathrm{S} 2-\mathrm{T}^{\mathrm{ab}}$ & $42.56 \pm 0.78^{\mathrm{a}}$ & $47.45 \pm 6.59^{\mathrm{a}}$ & $44.23 \pm 0.55^{\mathrm{a}}$ & $44.87 \pm 0.71^{\mathrm{ab}}$ & $47.22 \pm 1.13^{\mathrm{a}}$ & $46.30 \pm 1.29^{\mathrm{a}}$ \\
\hline $\mathrm{C}-\mathrm{H}^{\mathrm{ab}}$ & $43.23 \pm 1.19^{\mathrm{a}}$ & $46.65 \pm 3.71^{\mathrm{a}}$ & $48.11 \pm 7.91^{\mathrm{a}}$ & $47.30 \pm 0.96^{\mathrm{ab}}$ & $46.57 \pm 0.38^{a}$ & $45.27 \pm 0.93^{\mathrm{a}}$ \\
\hline $\mathrm{S} 1-\mathrm{H}^{\mathrm{ab}}$ & $42.08 \pm 0.98^{\mathrm{a}}$ & $46.02 \pm 2.31^{\mathrm{a}}$ & $44.98 \pm 0.92^{\mathrm{a}}$ & $47.84 \pm 1.15^{\mathrm{a}}$ & $46.00 \pm 0.79^{\mathrm{a}}$ & $46.55 \pm 0.64^{\mathrm{a}}$ \\
\hline $\mathrm{S} 2-\mathrm{H}^{\mathrm{a}}$ & $42.56 \pm 0.78^{a}$ & $46.67 \pm 0.99^{a}$ & $48.59 \pm 4.04^{\mathrm{a}}$ & $48.10 \pm 2.59^{\mathrm{a}}$ & $47.81 \pm 4.16^{\mathrm{a}}$ & $46.47 \pm 2.10^{\mathrm{a}}$ \\
\hline
\end{tabular}

* Data are the mean \pm standard deviation.

${ }^{\mathrm{a}, \mathrm{b}}$ Means in a column not having a common superscript letter are different $(p<0.05)$.

${ }^{\mathrm{A}, \mathrm{B}}$ Means in a row not having a common superscript letter are different $(p<0.05)$.

Table 10. Polyunsaturated fatty acid (PUFA) contents of fermented turkey sausages during $120 \mathrm{~d}$ storage*

\begin{tabular}{|c|c|c|c|c|c|c|}
\hline \multirow{2}{*}{ Groups } & \multicolumn{6}{|c|}{ Periods } \\
\hline & Initial mix ${ }^{\mathrm{A}}$ & $0^{\mathrm{B}}$ & $30^{\mathrm{B}}$ & $60^{\mathrm{B}}$ & $90^{\mathrm{B}}$ & $120^{\mathrm{B}}$ \\
\hline $\mathrm{C}-\mathrm{T}^{\mathrm{a}}$ & $26.70 \pm 0.62^{\mathrm{a}}$ & $23.95 \pm 0.92^{\mathrm{a}}$ & $24.76 \pm 1.32^{\mathrm{a}}$ & $24.62 \pm 0.99^{\mathrm{a}}$ & $26.88 \pm 0.06^{\mathrm{a}}$ & $22.95 \pm 0.65^{\mathrm{a}}$ \\
\hline $\mathrm{S} 1-\mathrm{T}^{\mathrm{a}}$ & $25.53 \pm 0.09^{\mathrm{b}}$ & $25.22 \pm 1.23^{\mathrm{a}}$ & $23.15 \pm 2.70^{\mathrm{a}}$ & $23.12 \pm 0.46^{\mathrm{a}}$ & $22.11 \pm 0.35^{\mathrm{ab}}$ & $22.61 \pm 1.76^{\mathrm{a}}$ \\
\hline $\mathrm{S} 2-\mathrm{T}^{\mathrm{a}}$ & $27.16 \pm 0.09^{\mathrm{a}}$ & $25.88 \pm 2.12^{\mathrm{a}}$ & $26.07 \pm 1.71^{\mathrm{a}}$ & $24.60 \pm 1.70^{\mathrm{a}}$ & $22.72 \pm 0.54^{\mathrm{ab}}$ & $22.33 \pm 0.93^{\mathrm{a}}$ \\
\hline $\mathrm{C}-\mathrm{H}^{\mathrm{a}}$ & $26.70 \pm 0.62^{\mathrm{a}}$ & $23.51 \pm 3.84^{\mathrm{a}}$ & $23.15 \pm 0.95^{\mathrm{a}}$ & $23.64 \pm 2.27^{\mathrm{a}}$ & $23.44 \pm 3.71^{\mathrm{ab}}$ & $25.75 \pm 2.36^{\mathrm{a}}$ \\
\hline $\mathrm{S} 1-\mathrm{H}^{\mathrm{a}}$ & $25.53 \pm 0.09^{\mathrm{b}}$ & $24.13 \pm 1.32^{\mathrm{a}}$ & $26.15 \pm 1.14^{\mathrm{a}}$ & $24.14 \pm 0.97^{\mathrm{a}}$ & $22.04 \pm 1.23^{\mathrm{b}}$ & $22.85 \pm 1.11^{\mathrm{a}}$ \\
\hline $\mathrm{S} 2-\mathrm{H}^{\mathrm{a}}$ & $27.16 \pm 0.09^{\mathrm{a}}$ & $25.17 \pm 0.51^{\mathrm{a}}$ & $24.05 \pm 3.09^{\mathrm{a}}$ & $23.85 \pm 2.11^{\mathrm{a}}$ & $23.46 \pm 2.20^{\mathrm{ab}}$ & $23.00 \pm 0.41^{\mathrm{a}}$ \\
\hline
\end{tabular}

* Data are the meantstandard deviation.

${ }^{\mathrm{a}, \mathrm{b}}$ Means in a column not having a common superscript letter are different $(p<0.05)$.

${ }^{\mathrm{A}, \mathrm{B}}$ Means in a row not having a common superscript letter are different $(p<0.05)$.

methodologies. For heated fermented sausages, docosahexaenoic acid content of $\mathrm{C}-\mathrm{H}$ and $\mathrm{S} 1-\mathrm{H}$ showed decreased at the end of heating stage $(p<0.05)$. The amounts of stearic, linoleic and arachidonic acids of $\mathrm{S} 2-\mathrm{H}$ group also decreased after heating stage $(p<0.05)$.

Considering storage periods, significant increase in the amount of palmitic acid and decrease in the amount of palmitoleic acid were noticed in traditionally processed C-T $(p<0.05)$. During storage, palmitoleic, stearic and arachidonic acids of S1-T decreased significantly $(p<0.05)$ while palmitic and arachidonic acids of S2-T showed significant increases $(p<0.05)$.

\section{Discussion}

For both traditionally and heat processed fermented sausages, the highest moisture content was measured in C-T and $\mathrm{C}-\mathrm{H}$ groups, while S1-T groups had the lowest moisture values (Table 1). In addition, utilizing heat treatment in comparison with traditionally processed fermented sausages resulted in lower moisture content. This difference is due to protein denaturation by $\mathrm{pH}$ values below 5.3 and heat treatment which resulted in decreases in water holding capacities of protein fractions (Acton and Dick, 1977; Acton and Keller, 1974; Korel, 1996). Therefore, no statistical difference in proximate composition was found in fermented sausages produced either by traditional or heated methods $(p>0.05)$.

The FFA values of initial mixes showed significant increases after fermentation stage ( $p<0.05$, Table 2, Table 3$)$. Many investigators also reported an increase in FFA contents of fermented sausages after fermentation stage and stated that this increase continued during ripening period as a result of endogenous and exogenous lipases (Casaburi et al., 2008; Chizzolini et al., 1998; Demeyer et al., 1974; Gandemer, 2002; Montel et al., 1993; Montel et al., 1998; Navarro et al., 1997). S2-T group had the highest FFA content S1-T had the lowest FFA content after drying stage ( $p>0.05$, Table 2). Stahnke (1995) indicated that $S$. xylosus and P. pentosaceus possesses lipolytic activity. Johansson et al. (1994) stated that, both organisms could be responsible for the lipolysis at the beginning of ripening, but only P. pentosaceus in the later stages. Johansson et al. (1994) stated that the free fatty acid content of fermented sausages increased during processing and reached $6.8 \%$ after $63 \mathrm{~d}$ which was lower than the findings of the current study. The lowest FFA value was measured in S1$\mathrm{T}$ group having the lowest $\mathrm{pH}$ and highest titratable acidity value after drying stage ( $p>0.05$, Table 2$)$. This could be explained by the decreasing effect of low $\mathrm{pH}$ value on the lipolytic activity of staphylococci (Demeyer et al., 2000; Hierro et al. 1997; Montel et al., 1998; Zanardi et 
al., 2004). The FFA values of all fermented sausage groups showed increases, especially after day $60(p<0.05$, Table 4). This increase was mainly due to the activity of muscle and adipose lipases at low water activity values (Hierro et al., 1997; Ordonez et al., 1999). Although heating stage resulted in a decreasing effect on both endogenous and microbial lipolytic activity, traditional style fermented sausages had higher FFA contents in comparison with the heat processed fermented sausage groups.

Thiobarbituric acid value constitutes a measurement of lipid oxidation. TBA values of initial mixes increased during ripening period ( $p<0.05$, Table 5). Marco et al. (2006) also reported increases in TBA values after fermentation and drying processes of dry fermented sausages. Heating stage resulted in a slight increase in TBA values of heat processed fermented sausages ( $p>0.05$, Table 6). Similar results stated by Coşkuner (2002) within the traditional and heat processed fermented beef sausages. Salgado et al. (2005) reported that TBA values were between 0.66$0.70 \mathrm{mg} \cdot \mathrm{kg}^{-1}$ in industrial Chorizo de cebolla, a Spanish traditional sausage. Lorenzo et al. (2000) also measured TBA value of Botillo, a Spanish traditional sausage as 0.96 $\mathrm{mg} \cdot \mathrm{kg}^{-1}$ sample which is higher than the measurements of current study. Although many investigators stated that TBA values of fermented sausages were affected by ripening time (Bozkurt and Erkmen, 2002; Karabacak and Bozkurt, 2008; Salgado et al., 2005) Visessanguan et al. (2006) reported a decrease in TBA values after fermentation. TBA values of fermented sausage groups produced by both methodologies did not show increases during the storage periods (Table 7). This could be explained by that malonaldehyde is a secondary product of lipid oxidation and these unstable changes are thought to be the result of malonaldehyde reactions with proteins and sugars (Ansorena and Asiasaran, 2004; Nassu et al., 2003). In this research starter culture utilizing resulted in lower TBA values in comparison with the control groups for both processing methodologies.

The MUFA displayed contents higher than PUFA and SFA in all the fermented sausage groups analyzed during ripening (Table 8). Similar results were also reported by Casaburi et al. (2008). Although the amount of MUFA increased after drying stage in all fermented sausage groups remained stable during storage periods $(p<0.05$, Table 9). The results indicated that PUFA contents of both traditionally and heat processed fermented sausages decreased at the stage of drying (Table 10) $(p<0.05$, Table 10). Gandemer (2002) stated that lipid oxidation resulted in a decrease in content of PUFA. In general, using starter cul- ture and heat application had no significant effect on the amounts of total SFA and PUFA ( $p>0.05)$, while C-T had the lowest MUFA content $(p<0.05)$. Galgano et al. (2003) also pointed out that during the fermentation stage; utilizing starter culture in manufacture of fermented sausages did not affect the amounts of SFA, MUFA and PUFA. Lipolytic activity at the beginning of fermentation was attributed to both lipases of muscular tissue and microbial origin (Toldra, 1998). However, Lactobacillus species are generally weakly lipolytic and also microbial lipases generally are very sensitive to $\mathrm{pH}$ (Montel et al., 1998). Several authors confirmed the importance of endogenous lipase activity in fermented meat products (Ansorena and Asiasaran, 2004; Bozkurt and Erkmen, 2002; Chizzolini et al., 1998; Molly et al., 1996).

It was seen that the main fatty acid was oleic (C 18:1) acid as previously reported by Samelis et al. (1993), Visessanguan et al. (2006) and Casaburi et al. (2008) and, followed by linoleic (C 18:2), palmitic (C 16:0), linolenic (C 18:3) and myristic (C 14:0) acids. In current study, palmitoleic (C 16:1), stearic (C 18:0), arachidonic (C 20:4) and docosahexaenoic (C 22:6) acids were also detected.

In conclusion, heating stage inhibited the lipolytic activity of both endogenous and exogenous lipases and increased the lipid oxidation in all heat processed fermented sausage groups. In addition, using starter culture mixes did have a significant effect on lipolytic changes such as TBA and FFA values during ripening and refrigerated storage for four months.

\section{Acknowledgements}

This study was supported by Ankara University Scientific Research Project Funding (BAP 2003-07-11-075).

\section{References}

1. Acton, J. C. and Dick, R. L. (1977) Cured color development during fermented sausage processing. J. Food Sci. 42, 895897.

2. Acton, J. C. and Keller, J. E. (1974) Effect of fermented meat pH on summer sausage properties. J. Milk Food Technol. 37, 570-576.

3. Ansorena, D. and Asiasaran, I. (2004) Effect of storage and packaging on fatty acid composition and oxidation in dry fermented sausages made with added olive oil and antioxidants. Meat Sci. 67, 237-244.

4. AOAC No 950.46. (2006) Moisture in meat. In: Horwitz, W. and Latimer, G. W. (Ed): Official Methods of Analysis of AOAC International. 18th. Edition. Maryland, 39, p. 1. 
5. AOAC No 928.08. (2006) Nitrogen in meat - Kjeldahl method. In: Horwitz, W. and Latimer, G. W. (Ed): Official Methods of Analysis of AOAC International. 18th. Edition. Maryland, 39, p. 5.

6. AOAC No 960.39. (2006) Fat (crude) or ether extract in meat. In: Horwitz, W. and Latimer, G. W. (Ed): Official Methods of Analysis of AOAC International. 18th. Edition. Maryland, 39, p. 2.

7. AOAC No 950.153. (2006) Ash of meat. In: Horwitz, W. and Latimer, G. W. (Ed): Official Methods of Analysis of AOAC International. 18th. Edition. Maryland, 39, p. 4.

8. AOAC No. 940.28. (2006) Fatty acids (free) in crude and refined oils. In: Horwitz, W. and Latimer, G. W. (Ed): Official Methods of Analysis of AOAC International. 18th. Edition. Maryland, 41, p. 12.

9. Bligh, E. G. and Dyer, W. J. (1959) A rapid method of total lipid extraction and purification. Can. J. Biochem. Phys. 37, 911-913.

10. Baggio, S. R., Vicente, E., and Bragagnolo, N. (2002) Cholesterol oxides, cholesterol, total lipid and fatty acid composition in turkey meat. J. Agric. Food Chem. 50, 5981-5986.

11. Bozkurt, H. and Erkmen, O. (2002) Effects of starter cultures and additives on the quality of Turkish style sausage (sucuk). Meat Sci. 61, 149-156.

12. Bozkurt, H. and Erkmen, O. (2007) Effects of some commercial additives on the quality of sucuk (Turkish dry-fermented sausage). Food Chem. 101, 1465-1473.

13. Casaburi, A., Aristoy, M. C., Cavella, S., Di Monaco, R., Ercolini, D., Toldra, F., and Villani, F. (2007) Biochemical and sensory characteristics of traditional fermented sausages of Vallo di Diano (Southern Italy) as affected by the use of starter culture. Meat Sci. 76, 295-307.

14. Casaburi, A., Di Monaco R., Cavella, S., Toldra, F., Ercolini, D., and Villani, F. (2008) Proteolytic and lipolytic starter cultures and their effect on traditional fermented sausages ripening and sensory traits. Food Microbiol. 25, 335-347.

15. Chizzolini, R., Novelli, E., and Zanardi, E. (1998) Oxidation in traditional Mediterranean meat products. Meat Sci. 49, 8799.

16. Coşkuner, Ö. (2002) Türk sucuğunda lipid oksidasyonuna ve serbest yaš asitleri olupumuna 1 sıl işlemin etkisi. M. Sc. thesis. Ankara Üniv., Ankara, Turkiye.

17. Çolak, H., Hampikyan, H., Ulusoy, B., and Bingöl, B. (2007) Presence of Listeria monocytogenes in Turkish style fermented sausage (sucuk). Food Control 18, 30-32.

18. Demeyer, D., Hooze, J., and Mesdom, H. (1974). Specifity of lipolysis during dry sausage ripening. J. Food Sci. 39, 293296.

19. Demeyer, D., Raemaekes, M., Rizzo, A., Holck, A., De Smedt, A., ten Brink, B., Hagen, B., Montel, C., Zanardi, E., Murbrekk, E., Leroy, F., Vandendriessche, F., Lorensten, K., Venema, K., Sunesen, L., Stahnke, L., De Vuyst, L., Talon, R., Chizzolini, R., and Eerola, S. (2000) Control of bioflavour and safety in fermented sausages: First results of European project. Food Res. Int. 33, 171-180.

20. Deumier, F. and Collignan, A. (2003) The effects of sodium lactate and starter cultures on $\mathrm{pH}$, lactic acid bacteria, Listeria monocytogenes and Salmonella spp. Levels in pure chicken dry fermented sausage. Meat Sci. 65, 1165-1174.

21. Drosinos, E. H., Mataragas, M., Xiraphi, N., Moschonas, G., Gaitis, F., and Metaxopoulos, J. (2005) Characterization of the microbial flora from a traditional Greek fermented sausage. Meat Sci. 60, 307-317.

22. Ensoy, Ü., Kolsarıcı, N., Candoğ an, K. and Karslıoğlu, B. (2010) Changes in biochemical and microbiological characteristics of turkey sucuks as affected by processing and starter culture utilization. J. Muscle Foods 21, 142-165.

23. Fernandez, M., Ordonez, J. A., Bruna, J. M., Herranz, B., and Hoz, L. (2000) Accelerated ripening of dry fermented sausages. Trends Food Sci. Tech. 11, 201-209.

24. Ferreira, V, Barbosa, J., Vendeiro, S., Mota, A., Silva, F., Monteiro, M. J., Hogg, T., Gibbs, P., and Teixeira, P. (2006) Chemical and microbiological characterization of alheira: A typical Portuguese fermented sausage with particular reference to factors relating to food safety. Meat Sci. 73, 570-575.

25. Ferreira, M. M. C., Morgano, M. A., Queiroz, S. C. N., and Mantovani, D. M. B. (2000) Relations of the minerals and fatty acid contents in processed Turkey meat products. Food Chem. 69, 259-265.

26. Galgano, F., Favati, F., Schirone, M., Martuscelli, M., and Crudelle, M. A. (2003) Influence of indigenous starter cultures on free fatty acids content during ripening in Artisan sausages produced in Basillicata region. Food Technol. Biotech. 41, 253-258.

27. Gandemer, G. (2002) Lipids in muscles and adipose tissues, changes during processing and sensory properties of meat products. Meat Sci. 62, 309-321.

28. Hierro, E., de la Hoz, L., and Ordonez, J. A. (1997) Contribution of microbial and meat endogenous enzymes to the lipolysis of dry fermented sausages. J. Agric. Food Chem. 45, 2989-2995.

29. IUPAC. (1987) Method Preparation of the fatty acid methyl esters. In: Standard methods for analysis of oils, fats and derivates. 7th ed. London, England: International Union of Pure and Applied Chemistry, Blackwell Scientific Publications, p. 2301.

30. Johansson, G., Berdague, J. L., Larsson, M., Tran, N., and Borch, E. (1994) Lipolysis, proteolysis and formation of volatile components during ripening of a fermented sausage with Pediococcus pentosaceus and Staphylococcus xylosus as starter cultures. Meat Sci. 38, 203-218.

31. Karabacak, S. and Bozkurt, H. (2008) Effects of Urtica dioica and Hibiscus sabdariffa on the quality and safety of sucuks (Turkish dry fermented sausage). Meat Sci. 78, 288-296.

32. Kenneally, P. M., Schwarz, G., Fransen, N. G., and Arenot, E. K. (1998) Lipolytic starter culture effects on production of free fatty acids in fermented sausages. J. Food Sci. 63, 538542.

33. Korel, F. (1996) Analyses of fermented sausages and the use of starer cultures and carbohydrate substrates in fermented turkey sausage. M.Sc. Thesis, Clemson University Clemson, USA. 
34. Lees, R. (1975) Food analysis. In: Lees R. (Ed): Analytical and quality control methods for the food manufacturer and buyer. 3th ed. London : Leonard Hill Books, pp. 245-246.

35. Leroy, F., Verluyten, J., and Vuyst, L. D. E. (2006) Functional meat starter cultures for improved sausage Fermentation. Int. J. Food Microbiol. 106, 270-285.

36. Lorenzo, J. M., Michinel, M., Lopez, M., and Carballo, J. (2000) Biochemical characteristics of two Spanish traditional dry-cured sausage varieties: Androlla and Botillo. J. Food Compos. Anal. 13, 809-817.

37. Lücke, F. K. (2000) Utilization of microbes to process and preserve meat. Meat Sci. 56, 105-115.

38. Marco, A., Navarro, J. L., and Flores, M. (2006) The influence of nitrite and nitrate on microbial, chemical and sensory parameters of slow dry fermented sausage. Meat Sci. 73, 660673.

39. Molly, K., Demeyer, D., Civera, T., and Veerplaetse, F. (1996) Lipolysis in a Belgian sausage: Relative importance of endogenous and bacterial enzymes. Meat Sci. 43, 235-244.

40. Montel, M. C., Masson, F., and Talon, R. (1998) Bacterial role in flavour development. Meat Sci. 49, 111-123.

41. Montel, M. C., Talon, R., Berdague, J. L., and Cantonnet, M. (1993) Effects of starter cultures on biochemical characteristics of French dry sausages. Meat Sci. 35, 229-240.

42. Nassu, R. T., Gonçalves, L. A. G., Silva, M. A. A. P., and Beserra, F. J. (2003) Oxidative stability of fermented goat meat sausage with different levels of natural antioxidant. Meat Sci. 63, 43-49.

43. Navarro, J. L., Nadal, M. I., Izquierdo, L., and Lores, J. (1997) Lipolysis in dry cured sausages as affected by processing conditions. Meat Sci. 45, 161-168.

44. Ordonez, J. A., Hierro, E. M., Bruna, J., and Hoz, L. (1999) Changes in the components of dry-fermented sausages during ripening. Crit. Rev Food Sci. 39, 329-367.

45. Rantsiou, K. and Cocolin, L. (2006) New developments in the study of the microbiota of naturally fermented sausage as determined by molecular methods: A review. Int. J. Food Microbiol. 108, 255-267.

46. Salgado, A., Garcia Fontan, M. C., Franco, I., Lopez, M., and
Carballo, J. (2005) Biochemical changes during the ripening of Chorizode cebolla a Spanish traditional sausage: Effect of the system of manufacture (homemade or industrial). Food Chem. 92, 413-424.

47. Samelis, J., Aggelis, G., and Metaxopoulos, J. (1993) Lipolytic and microbial changes during the natural fermentation and ripening of greek dry sausages. Meat Sci. 35, 371-385.

48. Soyer, A., Ertaş, A. H., and Üzümcüoğlu, Ü. (2005) Effect of processing conditions on the quality of naturally fermented Turkish sausages (sucuks). Meat Sci. 69, 135-141.

49. Stahnke, L. H. (1995) Dried sausages fermented with Staphylococcus xylosus at - Different temperatures and with different ingredient levels Part I. Chemical and bacteriological data. Meat Sci. 41, 179-191.

50. Talon, R., Walter, D., and Montel, M. C. (2000) Growth and effect of staphylococci and lactic acid bacteria on unsaturated free fatty acids. Meat Sci. 54, 41-47.

51. Tarladgis, B. G., Watts, B. M., Younathan, M. T., and Dugan, Tr. L. (1960) A distillation method for quantitative determination of malonaldehyde in rancid foods. J. Am. Chem. Soc. 37, 44-48.

52. Toldra, F. (1998) M. Proteolysis and lipolysis in flavour development of dry-cured meat products. Meat Sci. 49, 101-110.

53. Visessanguan, W., Benjakul, S., Riebroy, S., Yarchai, M., and Tapingkae, W. (2006) Changes in the lipid composition and fatty acid profile of Nham, a Thai fermented pork sausage, during fermentation. Food Chem. 94, 580-588.

54. Wong, M. K. and Sampugna, J. (1998) Moisture, total lipid, fatty acids, and cholesterol in raw ground Turkey. J.Agric. Food Chem. 41, 1229-1231.

55. Zanardi, E., Doigoni, V., Badiani, A., and Chizzolini, R. (2002) Lipid and colour stability of Milano-type sausages: Effect of packaging conditions. Meat Sci. 61, 7-14.

56. Zanardi, E., Ghidini, S., Battaglia, A., and Chizzolini, R. (2004) Lipolysis and lipid oxidation in fermented sausages depending on different processing conditions and different antioxidants. Meat Sci. 66, 415-423.

(Received 2013.10.10/Revised 2013.7.22/Accepted 2014.1.10) 